\title{
Connecting $\mathbf{R}$ with $\mathrm{D} 3$ for dynamic graphics, to explore multivariate data with tours
}

\author{
by Michael Kipp, Ursula Laa, Dianne Cook
}

\begin{abstract}
The tourr package in $\mathrm{R}$ has several algorithms and displays for showing multivariate data as a sequence of low-dimensional projections. It can display as a movie but has no capacity for interaction, such as stop/go, change tour type, drop/add variables. The tourrGui package provides these sorts of controls, but the interface is programmed with the dated RGtk2 package. This work explores using custom messages to pass data from R to D3 for viewing, using the Shiny framework. This is an approach that can be generally used for creating all sorts of interactive graphics.
\end{abstract}

\section{Introduction}

Did you know you can run any javascript you like in a Shiny application and you can pass whatever you want including JSON back and forth? This massively widens the scope of what you can do with Shiny, and generating a tour of multivariate data with this approach is a really good example of what is possible.

The tour algorithm (Asimov, 1985) is a way of systematically generating and displaying projections of high-dimensional spaces in order for the viewer to examine the multivariate distribution of data. It can do this either randomly, or by picking projections judged interesting according to some criterion or index function. The tourr package (Wickham et al., 2011) provides the computing and display in $\mathrm{R}$ to make several types of tours: grand, guided, little and local. The projection dimension can be chosen between one and the number of variables in the data. The display, though, has no capacity for interaction. The viewer can watch the tour like a movie, but not pause it and restart, or change tour type, or number of variables.

These interactive controls were provided with the tourrGui package (Huang et al., 2012), with was programmed with the RGtk2 package (Lawrence and Temple Lang, 2010). This is not the toolkit of choice today, and has been superceded with primarily web-capable tools, like Shiny (Chang et al., 2017). To display dynamic graphics though, is not straight-forward. This paper explains how to use D3 (Bostock et al., 2011) as the display engine in a Shiny graphical user interface (GUI), using custom message passing between server and client.

\section{Creating a tour, with the tourr package}

The tourr package (Wickham et al., 2011) is an R implementation of the tour algorithms discussed in Cook et al. (2007). It includes methods for geodesic interpolation and basis generation, as well as an implementation of the simulated annealing algorithm to optimise projection pursuit indices for the guided tour. The tour can be displayed directly in the R graphics device, for example, the code below generates a 1D density tour. Figure 1 shows snapshots.

library(tourr)

\# quartz() \# to display on a Mac; X11() \# For windows; The Rstudio graphics

\# device is not advised

animate_dist (flea[, 1:6], center $=$ TRUE)

A tour path is a smooth sequence of projection matrices, $p \times d$, that when combined with a matrix of $n$ data points, $n \times p$, and a rendering method, produces a steady stream of $d$-dimensional views of the data. Each tour is initialised with the new_tour () method, which instantiates a tour object and takes as arguments the data $X$, the tour method, e.g. guided_tour ( ), and the starting basis. Once initialised, a new target plane is chosen, and a series of steps along a geodesic path from starting to target plane are generated by interpolation.

This requires a series of calls to the tour object producing the series of projections. The steps are discrete, of size given by $\omega / \Delta$, where $\omega$ denotes the angular velocity of the geodesic interpolation, and $\Delta$ is a parameter denoting frames per second, reflecting the rendering speed of the device in use. The $\Delta$ parameter can be thought of as the frames per second, while $\omega$ affects the speed at which the tour moves through the projection space. For our purposes, $\Delta, \mathrm{fps}$ in the code, is set at 25 , while the $\omega$ can be adjusted by the user. 

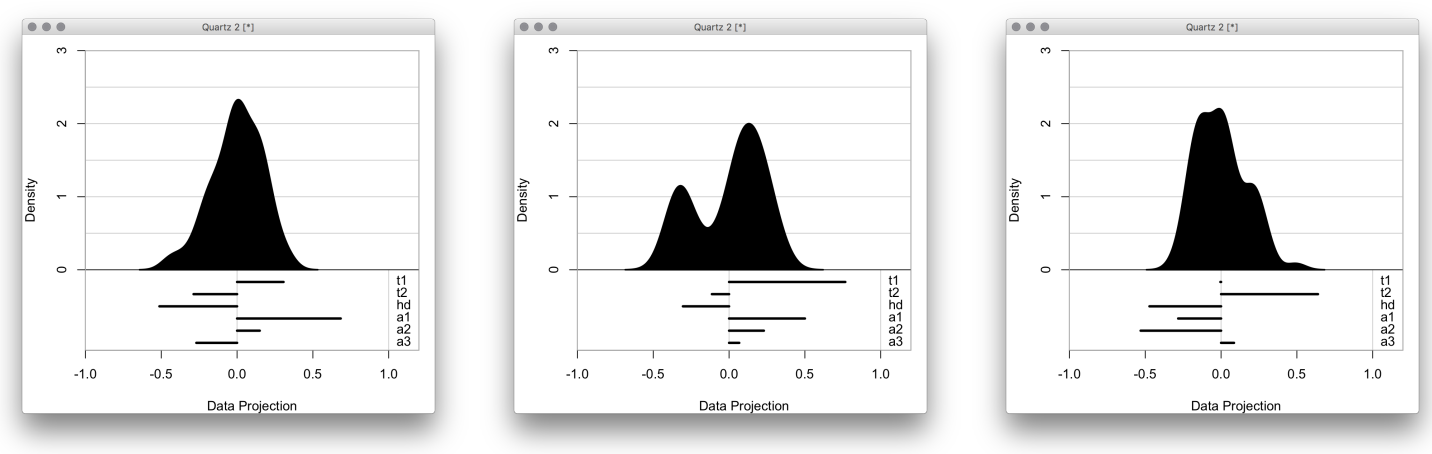

Figure 1: Three projections from a 1D tour of 6D data, displayed as a density. Full video can be seen at https://vimeo.com/255466661.

\section{Connecting the tour projections to D3 display using sendCustomMessage}

D3.js (Data-Driven Documents) (Bostock et al., 2011) is a JavaScript library for manipulating documents based on data. The advantages of D3 are similar to those provided by Shiny: namely, an industry standard with rich array of powerful, easy to use methods and widgets that can be displayed on a wide variety of devices, with a large user base. D3 works on data objects in the JavaScript Object Notation (JSON) format, which are then parsed and used to display customisable data visualisations.

The new implementation of the tour interface uses D3 to render each projection step returned by $\mathrm{R}$, focusing on $2 \mathrm{D}$ projections as a test case. It does this by drawing and re-drawing a scatterplot with dots (or circles in D3 language) and providing SVG objects for the web browser to render. Figure 2 shows the new GUI.

The Shiny functions session $\$$ sendCustomMessage() and Shiny. addCustomMessageHandler () are provided to transport data between $\mathrm{R}$ and JavaScript. Whenever the former is executed in $\mathrm{R}$, the latter function will execute a code block in JS. There are many examples of such functions being used to pass arbitrary data from an R app to a JS front-end, few examples exist of this basic functionality to update a D3 animation in real-time.

To set up the interface for the app, we need to load the relevant scripts into the Shiny app and assign a section for the resulting plots. This is done when setting up the user interface. We import D3 and our plotting code via the tags $\$$ script (for web links) and includescript (for reading from a full path). We use tags $\$$ div to assign an id for the output section that can be accessed in the D3 code.

tags $\$$ script (src = "https://d3js.org/d3.v4.min.js"),

includeScript (system.file("js/d3anim.js", package = "tourrGUID3")), tags\$div(id = "d3_output")

On the D3 side we can access the id defined in Shiny, and for example assign it to a scalable vector graphics (svg) object to be filled in D3 and rendered onto the Shiny app.

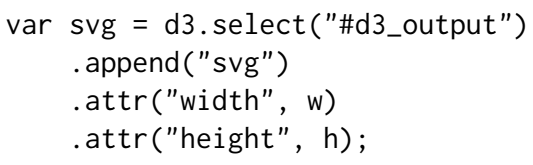

The data format expected by D3 is in JSON format, which combines two basic programming paradigms: a collection of name/value pairs, and an ordered list of values. R's preferred data formats include data frames, vectors and matrices. Every time a new projection has been calculated with the tour path, the resulting matrix needs to be converted to JSON and sent to D3. Using a named list we can send multiple JSON datasets to D3, e.g. to draw both the data points (stored in dataframe d) and the projection axes (stored in dataframe a). Converting dataframes will pass the column names to JSON. The code to send the D3 data looks like this:

session\$sendCustomMessage $($ type $=$ "data", message $=\operatorname{list}(d=\operatorname{toJSON}(d), a=\operatorname{toJSON}(a)))$

This code is from the observe environment from the server. $\mathrm{R}$ file. It converts the matrix of projected data points to JSON format, and sends it to JavaScript with the id data. The list entries of the "message" can parsed in D3 by its data() method, e.g. data(message.d) to access the projected data 


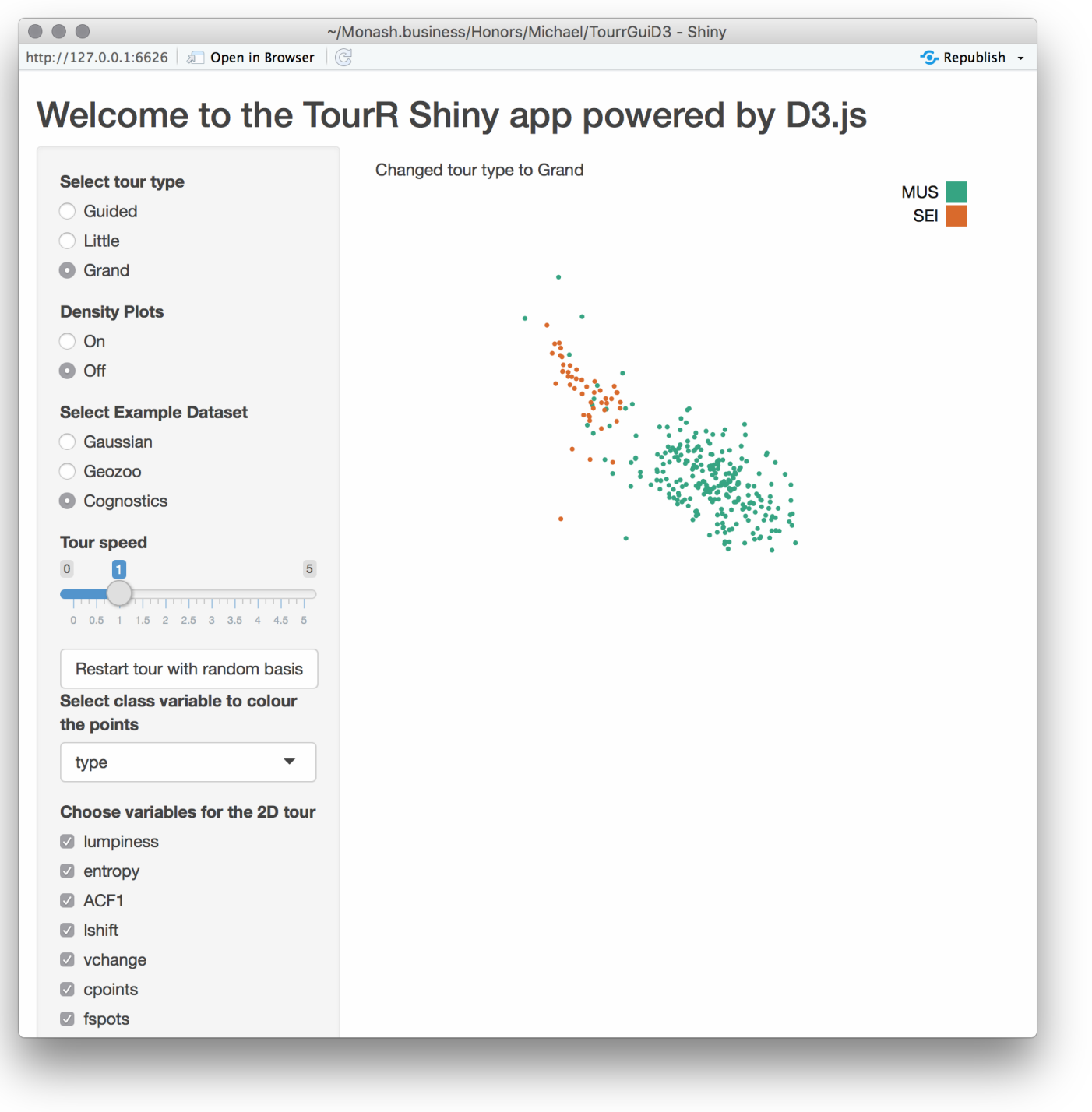

Figure 2: Shiny GUI for the tour, with D3 as the display engine. GUI provides controls to select tour type, change speed, restart, and select variables to include.

points, and we can access each column through the column names assigned in the original dataframe, and loop over all rows for rendering. All of the code required to render the scatterplots and legends, along with colours, is JavaScript code in the file d3anim. js. In particular, the data from $\mathrm{R}$ is handled with the following code:

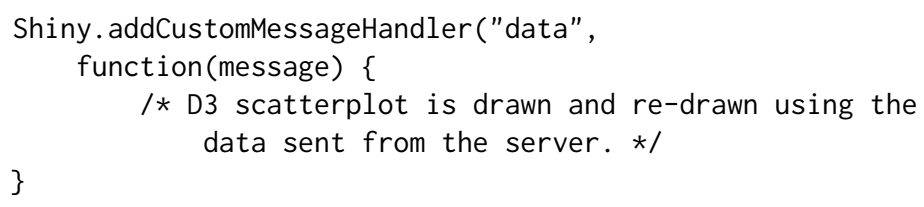

Every time the message is sent (25 times per second), the code-block is run.

\section{Getting projections}

The observeEvent Shiny method defines a code block to be run whenever some input value changes. The following code snippet restarts a tour using a random basis:

observeEvent (input\$restart_random, \{ 


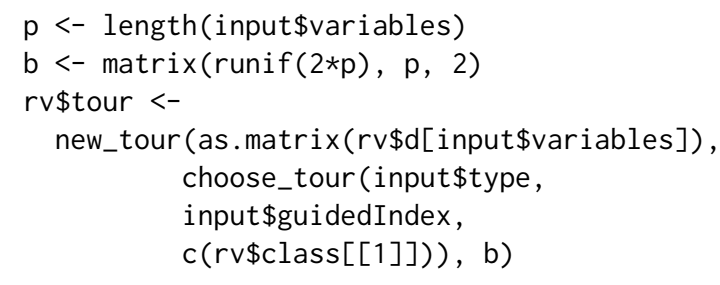

\})

The projections are calculated using the tour object in an observe() environment, which reexecutes the code whenever it is invalidated. The invalidation is either by a change in reactive value inside the code block, or we can schedule a re-execution by explicitly invalidating the observer after a selected interval using invalidateLater(). The projections are calculated using the following code block:

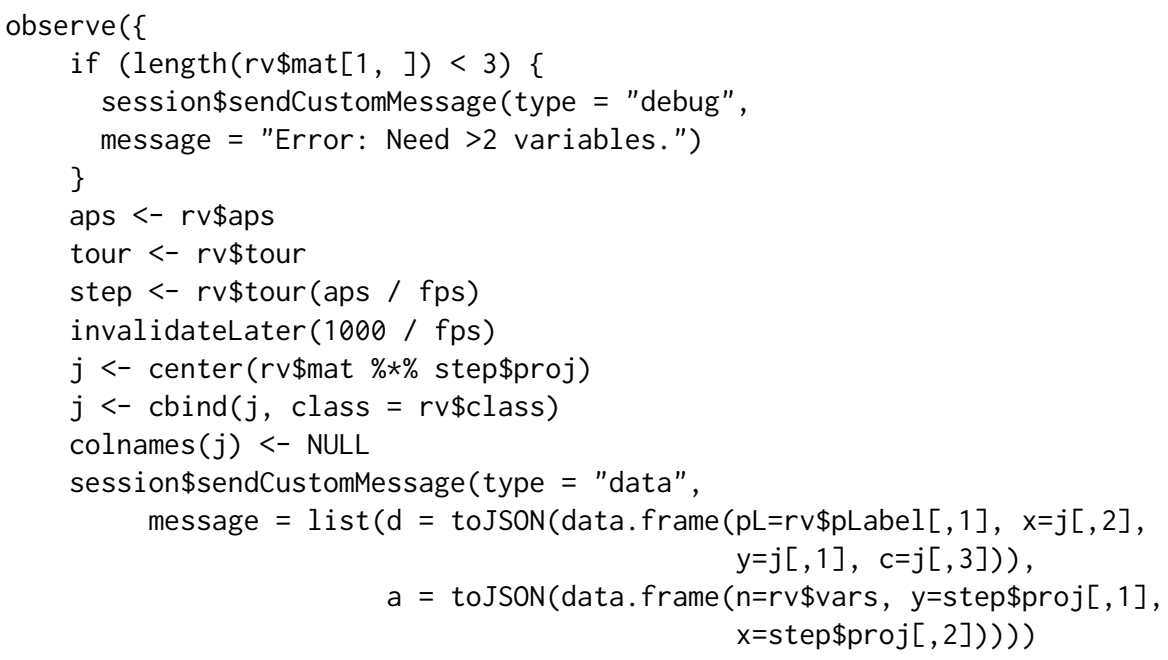

\section{Try it}

You can try the app yourself using this code:

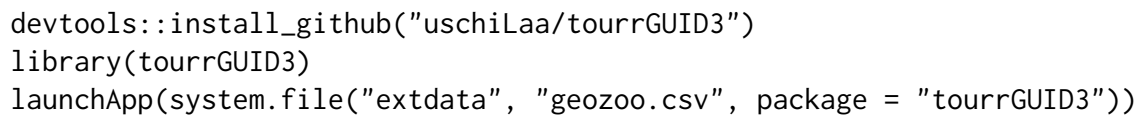

\section{Troubleshooting}

Fixing bugs in the JavaScript code can be cumbersome, as $\mathrm{R}$ and Shiny will not report any errors. Tracing JavaScript errors can be done when using the JavaScript console in the web browser. For example, in Google Chrome the console can be accessed via the "Developer Tools" option found under "Moore Tools" in the control menu. Typical errors that we encountered were version dependent syntax in D3, e.g. for axis definitions or scaling.

\section{Pros and cons}

The D3 canvas makes for smooth drawing and re-drawing of the data projections. Adding a GUI around the display is straightforward with the Shiny package, e.g. control elements such as stop/go, increase/decrease speed, change tour type, add/remove variables from the mix.

The main disadvantage is that the speed is inconsistent, as server and client play tag to keep up with each other, and the display cannot handle many observations. Noticeable slow down was oberved with 2000 points, the main reason being the rendering time required for the large number of SVG circle elements. The situation can be improved when using a single HTML5 canvas element to draw the scatter points, significantly reducing the rendering time.

Another disadvantage is that the displays needs to be coded anew. D3 provides mostly primitives, and example code, to make scatterplots, and contours, but the data displays all need to be coded again. 


\title{
Summary
}

The custom message tools from Shiny provide a way to share a tour path with the D3 renderer, and embed it in a Shiny GUI providing controls such as stop/go, increase/decrease speed, change tour type, add/remove variables. However, the approach doesn't provide the smooth motion that is needed for easy display of projections, and is slow for large numbers of observations.

\section{Code}

The code is available at https://github.com/uschiLaa/tourrGUID3, and the source material for this paper is available at https://github.com/dicook/paper-tourrd3.

\section{Acknowledgements}

Thanks to Yihui Xie for pointing out the custom message tools.

\section{Bibliography}

D. Asimov. The Grand Tour: A Tool for Viewing Multidimensional Data. SIAM Journal of Scientific and Statistical Computing, 6(1):128-143, 1985. URL https://doi .org/10.1137/0906011. [p1]

M. Bostock, V. Ogievetsky, and J. Heer. D3: Data-Driven Documents. IEEE Transactions on Visualization and Computer Graphics, 17(12):2301-2309, 2011. URL https://doi .org/10.1109/TVCG.2011.185. $[\mathrm{p} 1,2]$

W. Chang, J. Cheng, J. Allaire, Y. Xie, and J. McPherson. Shiny: Web Application Framework for R, 2017. URL https: //CRAN. R-project. org/package=shiny. R package version 1.0.5. [p1]

D. Cook, A. Buja, E. K. Lee, and H. Wickham. Grand Tours, Projection Pursuit Guided Tours and Manual Controls. Springer-Verlag, Berlin, Heidelberg, 2007. URL https://doi .org/10.1007/9783-540-33037-0_13. [p1]

B. Huang, D. Cook, and H. Wickham. tourrGui: A gWidgets GUI for the Tour to Explore HighDimensional Data Using Low-Dimensional Projections. Journal of Statistical Software, 49(6):1-12, 2012. URL https://doi.org/10.18637/jss.v049.i06. [p1]

M. Lawrence and D. Temple Lang. RGtk2: A Graphical User Interface Toolkit for R. Journal of Statistical Software, 37(8):1-52, 2010. URL https://doi.org/10.18637/jss.v037.i08. [p1]

H. Wickham, D. Cook, H. Hofmann, and A. Buja. tourr: An R Package for Exploring Multivariate Data with Projections. Journal of Statistical Software, 40(2):1-18, 2011. URL https: //doi . org/10.18637/ jss.v040.i02. [p1]

\author{
Michael Kipp \\ Monash University \\ Department of Econometrics and Business Statistics \\ mkipp271@gmail.com \\ Ursula Laa \\ Monash University \\ School of Physics and Astronomy \\ ursula.laa@monash.edu \\ Dianne Cook \\ Monash University \\ Department of Econometrics and Business Statistics \\ dicook@monash. edu
}

